\title{
Broadening Pfam Sequence Annotations
}

\author{
Jaina Mistry, Penny Coggill, Sean Eddy§, Rob Finn, John Tate, Alex Bateman \\ Wellcome Trust Sanger Institute, Wellcome Trust Genome Campus, Hinxton, Cambs, CB10 1SA, UK \\ §Janelia Farm Research Campus, Howard Hughes Medical Institute, 19700 Helix Drive, Asburn, VA, US
}

\section{What is Pfam?}

Pfam is a database, of conserved protein families or domains, commonly used for proteome annotation and sequence classification. It comprises two parts: (1) Pfam-A families, which are manually annotated, and consist of a representative seed alignment, hidden Markov models (HMMs), and a full alignment of all sequences that score above the curated threshold; and (2) Pfam-B families, automatically generated clusters of similar sequence regions not matched by Pfam-A that often indicate the presence of a domain. Many of the Pfam-A families are arranged into a hierarchical classification, termed clans. You can access and download the Pfam data via the website at http://pfam.sanger.ac.uk

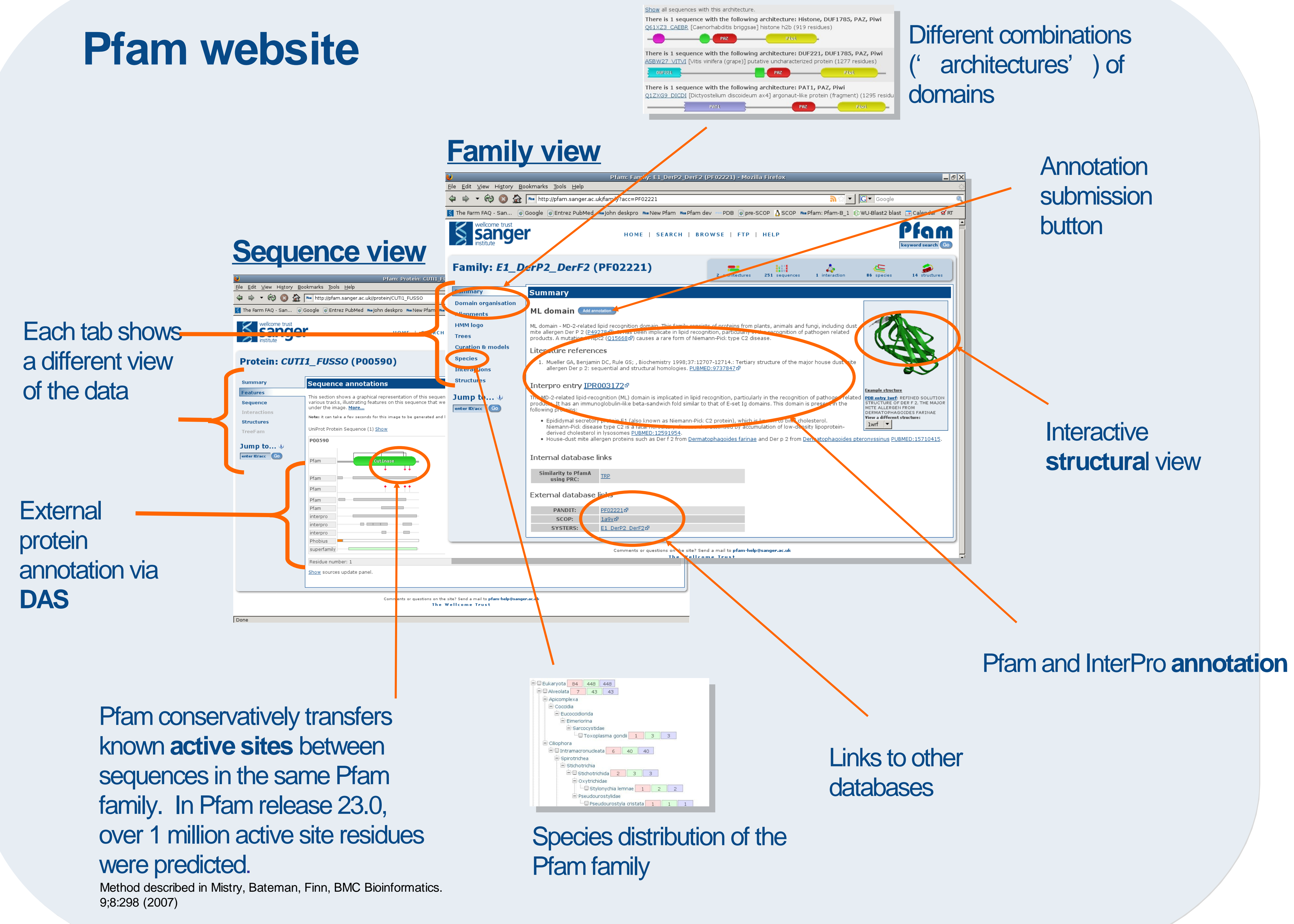

\section{Pfam coverage of proteomes}

The proteome coverage of Pfam varies between species. Coverage is typically measured in the following ways:

Sequence coverage is defined as the proportion of sequences that have a match to at least one Pfam-A family

Amino acid coverage is defined as the proportion of amino acids that belong to a Pfam-A family

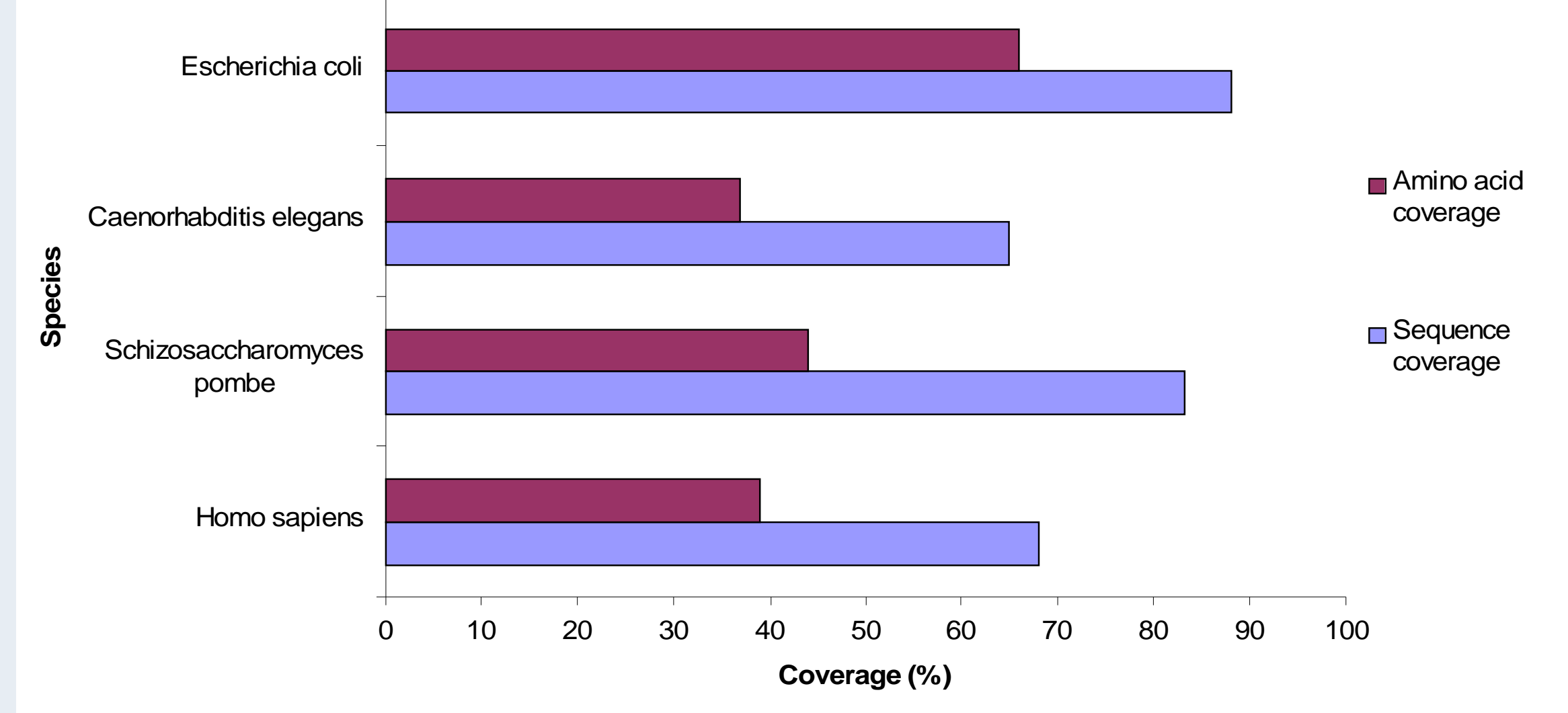

The coverage of a few model organisms is shown above. We achieve a much higher sequence coverage than amino acid coverage, and our coverage of bacterial proteomes is better than for other species.

\section{Towards a complete classification of protein space}

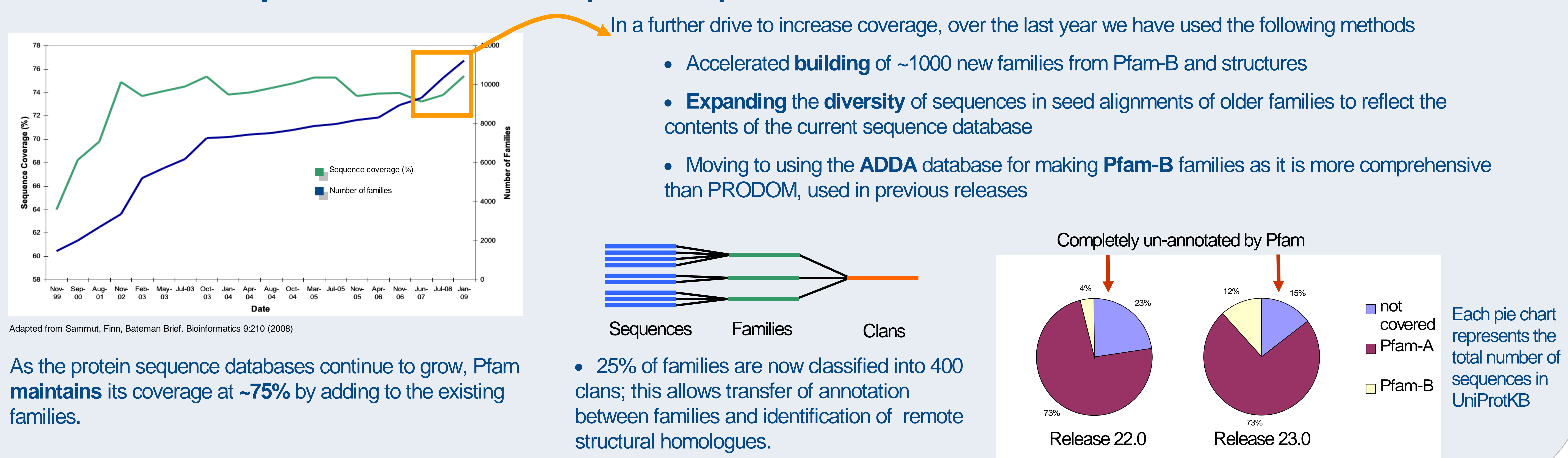

\section{Interacting with our community}

We support our user-community and receive feedback in the following ways:

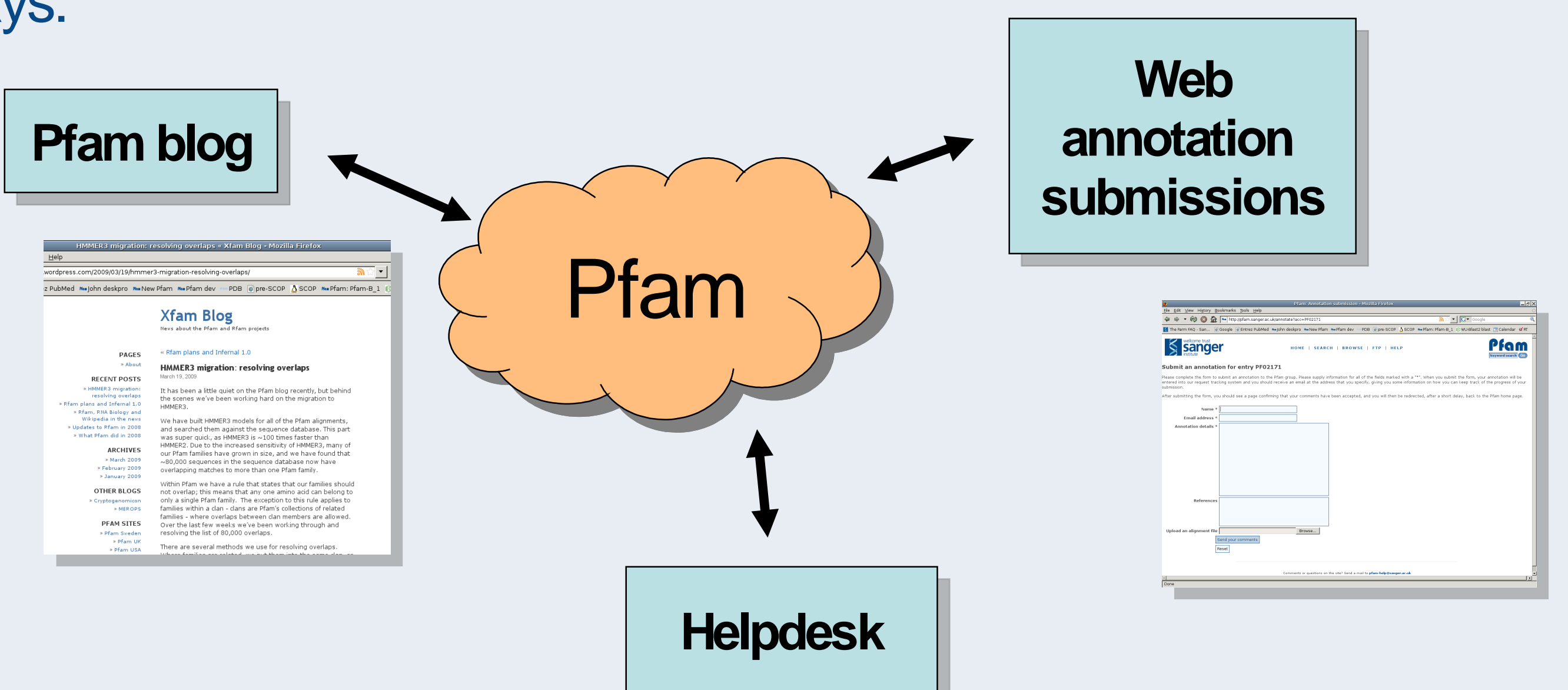

We welcome receipt of alignments, annotation and references for new families, and annotation-updates on existing families. All incoming queries to our helpdesk pfam-help@sanger.ac.uk are tracked.

Our blog informs users about Pfam news and future plans. It is linked from the Pfam website, or you can visit it at

http://xfam.wordpress.com

\section{Improved speed and sensitivity with HMMER3}

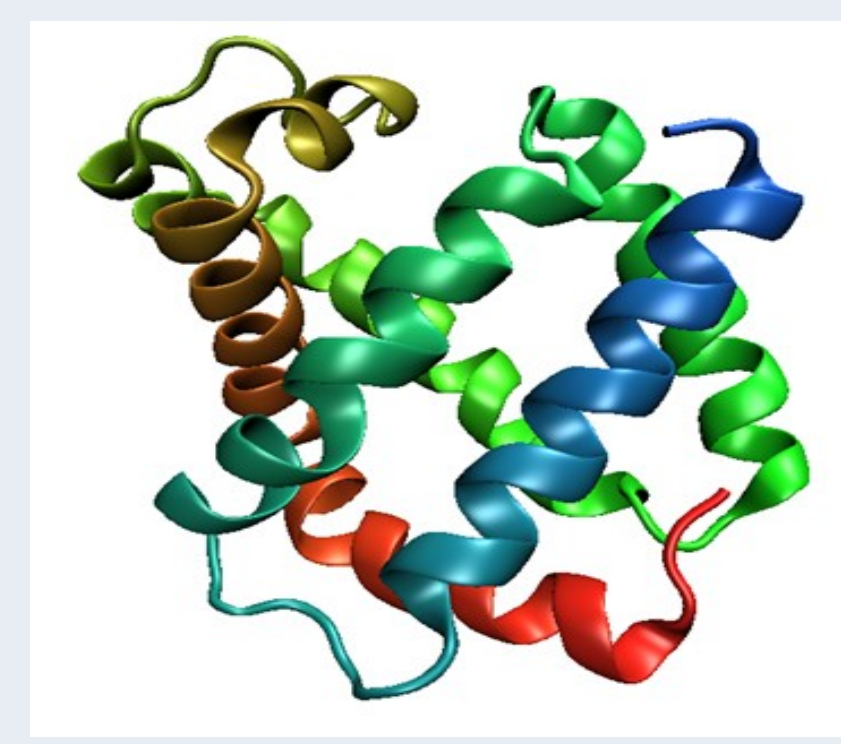
Aplysia myoglobin
(PDB 1mba) Wikipedia

With a cut-off at $E<=0.01$

(in $9 \mathrm{sec}$ )

HMMER3 finds 1002 globins (in $10 \mathrm{sec}$ )

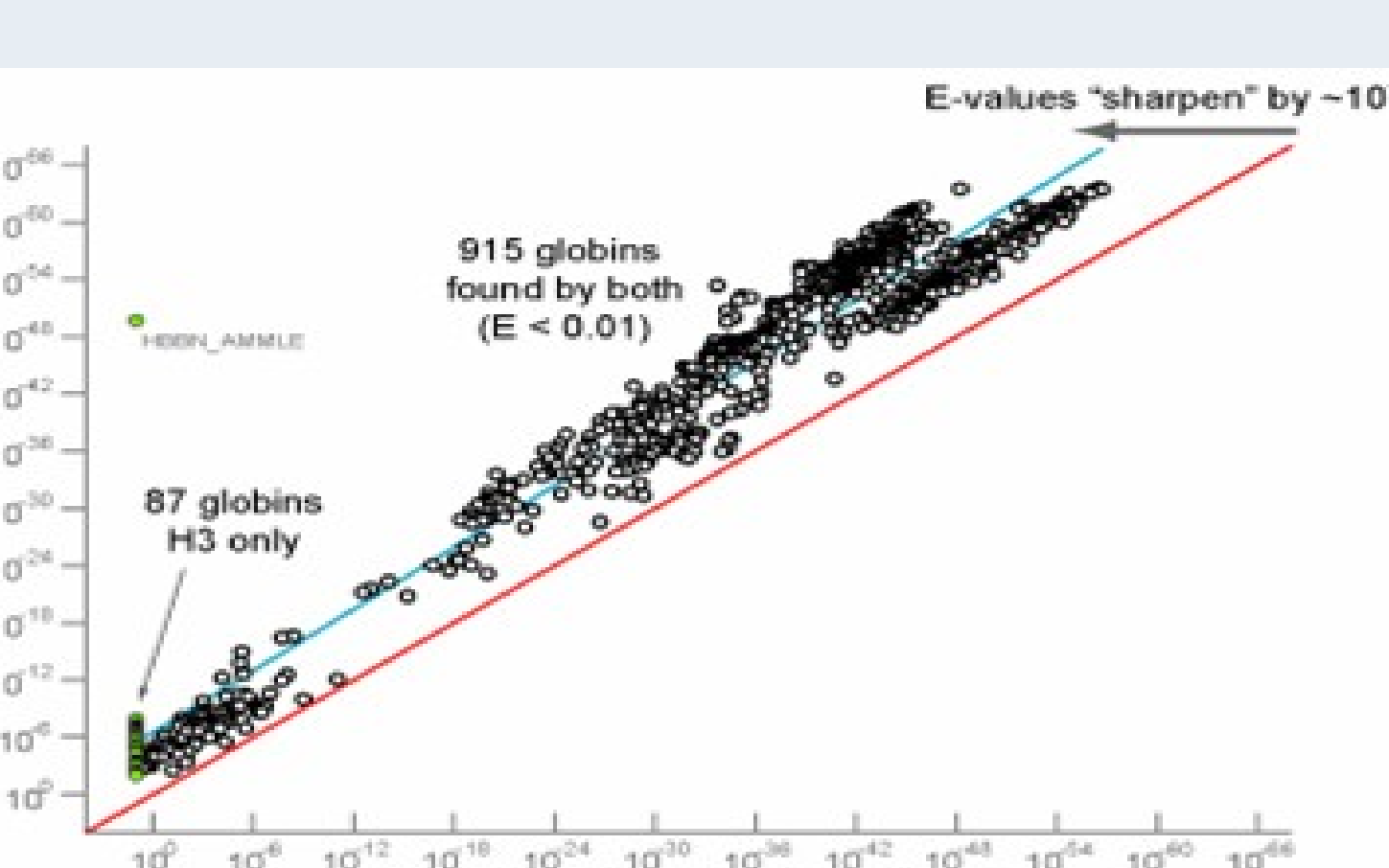

An initial profile-HMM was made from three vertebrate hemoglobins and one myoglobin using HMMER3 hmmbuild.

The HMM was searched with HMMER3 hmmsearch against Uniprot 7.0 (207K seqs, containing $~ 1060$ known globins). The results were compared with a PSI-BLAST search, starting with the same four sequences.

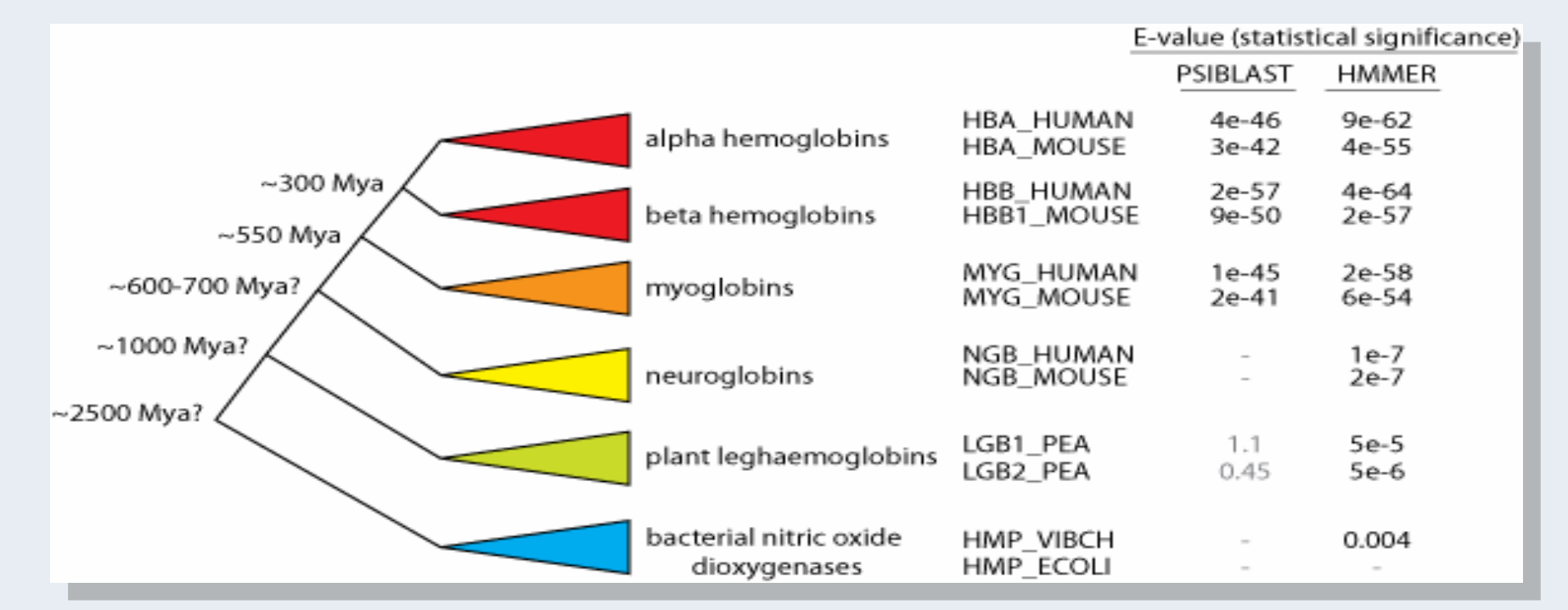

HMMER3 is more sensitive than PSI-BLAST in finding more distant relatives, and is 100 times faster than HMMER2 